\title{
Echoes of a Hidden Valley at Hadron Colliders
}

\author{
Matthew J. Strassler and Kathryn M. Zurek \\ Department of Physics, P.O Box 351560, \\ University of Washington, Seattle, WA 98195
}

\begin{abstract}
We consider examples of "hidden-valley" models, in which a new confining gauge group is added to the standard model. Such models often arise in string constructions, and elsewhere. The resulting (electrically-neutral) bound states can have low masses and long lifetimes, and could be observed at the LHC and Tevatron. Production multiplicities are often large. Final states with heavy flavor are common; lepton pairs, displaced vertices and/or missing energy are possible. Accounting for LEP constraints, we find LHC production cross-sections typically in the 1-100 fb range, though they can be larger. It is possible the Higgs boson could be discovered at the Tevatron through rare decays to the new particles.
\end{abstract}

As the era of the Large Hadron Collider (LHC) approaches, and the Tevatron accumulates data, it is important to consider well-motivated particle physics models that present novel and challenging experimental signals. There has been much recent work along these lines [1, 2, 3, 4, [5].

Here we discuss a class of "hidden-valley" models that has received little study. These are models in which the standard model (SM) gauge group $G_{S M}$ is extended by a non-abelian group $G_{v}$. All SM particles are neutral under $G_{v}$, but there are new light particles ("v-particles") charged under $G_{v}$ and neutral under $G_{S M}$. Higher dimension operators at the $\mathrm{TeV}$ scale (induced perhaps by a $Z^{\prime}$ or by a loop of heavy particles carrying both $G_{S M}$ and $G_{v}$ charges) allow interactions between SM fields and the new light particles. In this circumstance, the v-particles are rarely produced at LEPI or LEPII, but may be abundantly produced at the LHC, and perhaps even the Tevatron. In a confining hidden-valley model, all v-particles assemble themselves into $G_{v}$-neutral "vhadrons." Some of the v-hadrons can then decay, again via higher dimension operators, to gauge-invariant combinations of SM particles, with observable lifetimes. The diverse masses and lifetimes of the v-hadrons, their multiplicities, and the variety of possible final states make the v-phenomenology complex, and sensitive to underlying parameters, such as v-quark masses.

The hidden-valley scenario is consistent with data and is well motivated; it arises in many top-down models, including string-theory constructions [ 6$]$. It appears consistent with most methods for solving the hierarchy problem (supersymmetry, little Higgs models, TeV extra dimensions, Randall-Sundrum scenarios) so the vphenomenology outlined below may accompany more familiar physics associated with each of these solutions. However, the purpose of this paper is not model-building, but rather to call attention to generic signals which may not yet have been fully explored at the Tevatron or in studies for the LHC.

As there is no clearly "minimal" hidden-valley model, we will present a simple theory exhibiting many phenomena typical of a v-sector, which include the following:

- There are several long-lived v-hadrons, with masses typically of order the v-confinement scale $\Lambda_{v}$.

- Some v-hadrons may be stable, providing dark matter candidates and missing energy signals, while others decay to neutral combinations of SM particles.

- Decay lifetimes can vary over many orders of magnitude; v-hadrons may decay promptly, or produce a displaced vertex anywhere in the detector, or typically decay outside the detector.

- Some v-hadrons decay preferentially to heavy flavor, while others decay more democratically to $f \bar{f}$ final states ( $f$ any SM fermion) or to $f \bar{f}$ plus another v-hadron; other final states can include two or three gluons, $W W$ or $Z Z$. - V-hadron production multiplicities at the LHC may be large, especially if $\Lambda_{v} \ll 1 \mathrm{TeV}$.

A Simple v-Model: To the SM, we add a $U(1) \times S U\left(n_{v}\right)$ gauge group, with couplings $g^{\prime}$ and $g_{v}$; we omit the special case $n_{v}=2$. The $S U\left(n_{v}\right)$ interaction confines at the scale $1 \mathrm{GeV}<\Lambda_{v}<1 \mathrm{TeV}$. The $U(1)$ is broken by a scalar expectation value $\langle\phi\rangle$, giving a $Z^{\prime}$ of mass $\sim 1-6 \mathrm{TeV}$. We add two v-quark flavors $U, \bar{U}$ and $C, \bar{C}$ in the $n_{v}$ and $\overline{n_{v}}$ representation, and three righthanded neutrinos $N_{i}$; all become massive by coupling to $\phi$. These masses and anomaly cancellation restrict the $U(1)$ charges to those shown in table \ with $q_{+}$arbitrary and $q_{+}+q_{-}=-2$. (We omit the special case $q_{+}=q_{-}=-1$.) For definiteness, we take the SM particles (and the $N_{i}$ ) to be charged as under the usual $U(1)_{\chi}$ subgroup of $S O(10)$ that commutes with $G_{S M}$; see 7 . We impose a $Z_{2}$ symmetry forbidding Dirac neutrino masses, making the $N_{i}$ stable (and dark matter candidates.) Kinetic mixing between $U(1)_{\chi}$ and hypercharge,

\begin{tabular}{||c||c|c|c|c|c|c||c|c|c|c||c|c||}
\hline & $q_{i}$ & $\bar{u}_{i}$ & $\bar{d}_{i}$ & $\ell_{i}$ & $e_{i}^{+}$ & $N_{i}$ & $U$ & $\bar{U}$ & $C$ & $\bar{C}$ & $H$ & $\phi$ \\
\hline$S U(3)$ & $\mathbf{3}$ & $\overline{\mathbf{3}}$ & $\overline{\mathbf{3}}$ & $\mathbf{1}$ & $\mathbf{1}$ & $\mathbf{1}$ & $\mathbf{1}$ & $\mathbf{1}$ & $\mathbf{1}$ & $\mathbf{1}$ & $\mathbf{1}$ & $\mathbf{1}$ \\
\hline$S U(2)$ & $\mathbf{2}$ & $\mathbf{1}$ & $\mathbf{1}$ & $\mathbf{2}$ & $\mathbf{1}$ & $\mathbf{1}$ & $\mathbf{1}$ & $\mathbf{1}$ & $\mathbf{1}$ & $\mathbf{1}$ & $\mathbf{2}$ & $\mathbf{1}$ \\
\hline$U(1)_{Y}$ & $\frac{1}{6}$ & $-\frac{2}{3}$ & $\frac{1}{3}$ & $-\frac{1}{2}$ & 1 & 0 & 0 & 0 & 0 & 0 & $\frac{1}{2}$ & 0 \\
\hline$U(1)_{\chi}$ & $-\frac{1}{5}$ & $-\frac{1}{5}$ & $\frac{3}{5}$ & $\frac{3}{5}$ & $-\frac{1}{5}$ & -1 & $q_{+}$ & $q_{-}$ & $-q_{+}$ & $-q_{-}$ & $\frac{2}{5}$ & 2 \\
\hline$S U\left(n_{v}\right)$ & $\mathbf{1}$ & $\mathbf{1}$ & $\mathbf{1}$ & $\mathbf{1}$ & $\mathbf{1}$ & $\mathbf{1}$ & $\mathbf{n}_{\mathbf{v}}$ & $\overline{\mathbf{n}_{\mathbf{v}}}$ & $\mathbf{n}_{\mathbf{v}}$ & $\overline{\mathbf{n}_{\mathbf{v}}}$ & $\mathbf{1}$ & $\mathbf{1}$ \\
\hline
\end{tabular}

TABLE I: Charge assignments for the model before removal of kinetic mixing; $q_{+}+q_{-}=-2$. 
via the interaction $\frac{1}{2} k F_{\mu \nu}^{\prime} F_{Y}^{\mu \nu}$, cannot be forbidden; we treat $k$ as a free parameter. We may remove $k$ by a field redefinition, at the cost of changing the charges. In our formulas below, all factors of $g^{\prime}$ and all $U(1)_{\chi}$ charges $Q_{i}$ are defined after removal of $k$ by field redefinition; the new charges differ from those in Table \by $Q_{\chi}=Q_{\chi}^{0}-\left(k g_{1} / g^{\prime}\right) Y$ and, as expected [8], are no longer quantized. Some other classic $U(1)$ groups, such as $B-L$, are obtained for special values of $k$.

Two Light Flavors (2LF): We first consider the regime $m_{U} \sim m_{C} \ll \Lambda_{v}$, where the physics resembles that of QCD (Fig. 1). An approximate v-isospin between $U$ and $C$ quarks controls the spectrum; by analogy we call the (neutral!) v-pions $\pi_{v}^{ \pm}, \pi_{v}^{0}$. All v-hadrons decay rapidly to v-pions and v-nucleons, of which all are stable and invisible except the $\pi_{v}^{0}$. The $\pi_{v}^{0}$ is unique: with v-quark wave function $U \bar{U}-C \bar{C}$, it can decay via $Q \bar{Q} \rightarrow Z^{\prime} \rightarrow$ $f \bar{f}$, where $Q=U, C$ and $f$ is any SM fermion. Using $Q_{f}+Q_{\bar{f}}= \pm Q_{H}$ for all $f$, and $q_{+}+q_{-}=-Q_{\phi}$, we find (except near $m_{\pi_{v}} \sim m_{Z}$, where $\Gamma_{Z}$ must be included),

$$
\Gamma_{\pi_{v}^{0}}=\frac{1}{8 \pi} \frac{g^{\prime 4}}{m_{Z}^{\prime 4}} \frac{Q_{\phi}^{2} Q_{H}^{2} f_{\pi_{v}}^{2} m_{\pi_{v}}^{5}}{\left(m_{\pi_{v}}^{2}-m_{Z}^{2}\right)^{2}} \sum_{f} N_{c}^{f} m_{f}^{2} v_{f} .
$$

Here $m_{f}, v_{f}$ are the mass and velocity of $f ; N_{c}^{f}=3$ for quarks ( 1 for leptons). (For very small $m_{\pi_{v}}$ or $Q_{H}$, a loop effect may be dominant.) The extra factor of $\left(m_{\pi_{v}} / m_{Z}\right)^{4}$ at low $m_{\pi_{v}}$ arises because the axial currents for $Q$ and $f$ decouple at low momentum. Heavy flavor is favored, due to the same helicity-flip that enhances $\mu^{+} \nu$ in usual $\pi^{+}$decay. If $2 m_{b}<m_{\pi_{v}}<2 m_{t}$, the dominant decay is $\pi_{v} \rightarrow b \bar{b}$. For $m_{\pi_{v}} \gg m_{Z}$

$$
\Gamma_{\pi_{v} \rightarrow b \bar{b}} \sim 3 \times 10^{15} \sec ^{-1} \frac{f_{\pi_{v}}^{2} m_{\pi_{v}}}{(200 \mathrm{GeV})^{3}}\left(\frac{10 \mathrm{TeV}}{m_{Z^{\prime}} / g^{\prime}}\right)^{4},
$$

while for $m_{\pi_{v}} \ll m_{Z}$

$$
\Gamma_{\pi_{v} \rightarrow b \bar{b}} \sim 6 \times 10^{9} \sec ^{-1} \frac{f_{\pi_{v}}^{2} m_{\pi_{v}}^{5}}{(20 \mathrm{GeV})^{7}}\left(\frac{10 \mathrm{TeV}}{m_{Z^{\prime}} / g^{\prime}}\right)^{4} .
$$

(Here and elsewhere, we show explicit numbers for the simple case $n_{v}=3, k=0, q_{+}=-2$.) We will see (Fig. (2) that $m_{Z^{\prime}} / g^{\prime}$ can range widely, as can $m_{\pi_{v}}$ and $f_{\pi_{v}}$. Consequently $\pi_{v}^{0}$ decay may be prompt, or may occur with a displaced vertex in any element of the detector.

One Light Flavor (1LF): The regime $m_{C} \gg \Lambda_{v} \gg m_{U}$ is unfamiliar; its spectrum and decay modes are not precisely known. Multiple v-hadrons are stable against vstrong decays. For any $n_{v}$, these include both the lightest pseudoscalar $\eta_{v}^{\prime}$ and the lightest vector $\omega_{v}$ (if $\mathrm{C}$ is conserved.) The axial anomaly ensures $m_{\eta_{v}^{\prime}} / m_{\omega_{v}} \propto \sqrt{1 / n_{v}}$ [9]. Therefore, for small $n_{v}$, there will be other narrow resonances, including the scalar $\sigma_{v}$ (which is wide in $\mathrm{QCD}$,) the first excited v-baryons $\Delta_{v}^{*}$, and the vector $C \bar{U}$ meson (which we call $D_{v}^{*}$ by analogy with charm mesons.) For $n_{v}=3$, approximate mass degeneracies inherited from supersymmetry, analogous to the conjecture in [10], can be rigorously demonstrated [11] to be

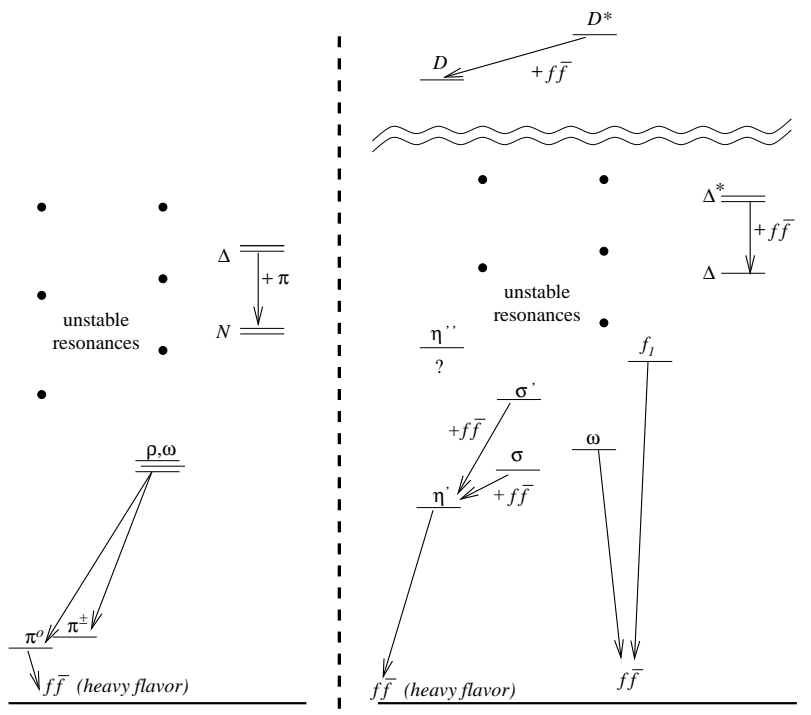

FIG. 1: Partial spectrum and decay modes in the two-lightflavor regime (left) and one-light-flavor regime (right); the latter is partly guesswork.

applicable [12]. It is expected [13] that the $m_{\eta_{v}^{\prime}}$ and $m_{\sigma_{v}}$ differ by no more than 30 percent; the $\omega_{v}$ is similarly accompanied by a $J^{P}=0^{+}$state $\sigma_{v}^{\prime}$, while the $f_{1 v}$ has a partner $0^{-}$state $\eta_{v}^{\prime \prime}$. Other states may be unstable.

For definiteness we assume the $\eta_{v}^{\prime}$ is the lightest vhadron (Fig. (1). The $\eta_{v}^{\prime}$ decay is similar to that of the $\pi_{v}^{0}$; the formulas (1)-(3) apply with simple adjustments. The $\omega_{v}$ decays, via $U \bar{U} \rightarrow Z^{\prime} \rightarrow f \bar{f}$, are typically prompt; ignoring $m_{f}$ and with $m_{\omega_{v}}-m_{Z}$ not too small,

$$
\begin{aligned}
\Gamma_{\omega_{v}} & \sim \frac{1}{96 \pi} \frac{g^{\prime 4}}{m_{Z^{\prime}}^{4}}(\Delta q)^{2} A^{2} m_{\omega_{v}}^{5} \sum_{f} N_{c}^{f} K\left(Q_{f}, Q_{\bar{f}}, \frac{m_{\omega_{v}}}{m_{Z}}\right) \\
& \sim 4 \times 10^{18} \sec ^{-1}\left(\frac{m_{\omega_{v}}}{200 \mathrm{GeV}}\right)^{5}\left(\frac{10 \mathrm{TeV}}{m_{Z^{\prime}} / g^{\prime}}\right)^{4},
\end{aligned}
$$

where in the last line we took $A^{2} \equiv\left|\left\langle\mathbf{0}\left|J_{U}^{\mu}+J_{\bar{U}}^{\mu}\right| \omega_{v}\right\rangle\right|^{2}=1$ and $\Delta q=q_{+}-q_{-}=-2$. The function $K$ includes effects of $Z-Z^{\prime}$ mixing; it has a resonance near $m_{Z}$ and for large $m_{\omega_{v}}$ goes to $Q_{f}^{2}+Q_{\bar{f}}^{2}$. For $k=0$ and $m_{\omega_{v}}<2 m_{t}, 2 m_{N}$, $\sum N_{c}^{f} K \approx 6(9)$ and $\operatorname{Br}\left(\omega_{v} \rightarrow \mu^{+} \mu^{-}\right) \sim 6 \%$ (3\%) for $m_{\omega_{v}} \gg m_{Z}\left(m_{\omega_{v}} \ll m_{Z}\right)$. (Decays to $W^{+} W^{-}$can occur through $Z^{\prime}-Z$ mixing; the branching fraction, while small, may be experimentally interesting.) A metastable $f_{1 v}$ decays similarly. Other v-hadrons such as $\sigma_{v}, \sigma_{v}^{\prime}, \Delta_{v}^{*}$ and $D_{v}^{*}$ most likely have three-body decays to $f \bar{f}$ plus a lighter v-hadron (Fig. 11); note the $\Delta_{v}$ and $D_{v}$ are stable. These decays are sensitive to small mass splittings. Due to unknown v-hadronic form factors, etc., lifetimes can 
only be estimated:

$$
\begin{aligned}
\Gamma & \sim \frac{1}{60 \pi^{3}} \frac{g^{\prime 4}}{m_{Z^{\prime}}^{4}}(\Delta m)^{5} \sum N_{c}^{f} K\left(Q_{f}, Q_{\bar{f}}, \frac{\Delta m}{m_{Z}}\right) \\
& \sim 3 \times 10^{14} \sec ^{-1}\left(\frac{\Delta m}{50 \mathrm{GeV}}\right)^{5}\left(\frac{10 \mathrm{TeV}}{m_{Z^{\prime}} / g^{\prime}}\right)^{4}
\end{aligned}
$$

where $\Delta m$ is the mass-difference between the initial and final v-hadron. Finally, although $D_{v}$ is invisible, $C \bar{C}$ onium states decay by annihilation to light, visible vhadrons; these decays would allow access to $m_{C}$.

Unstable $C$ : In the model above, the $C$ is stable, but many variants of this model naturally have flavorchanging interactions that cause $C$ decays. These could include higher dimension operators such as $C \bar{U} U \bar{U}$, induced at tree level (as would occur if there are two scalars $\phi_{1}, \phi_{2}$ instead of one) or by loops (as with vsquark masses in a supersymmetric variant of the model.) Rather than explore models here, we may view this as introducing one more free parameter: the lifetime for the lightest $C \bar{U}$ v-meson(s). In the $1 \mathrm{LF}$ regime, the $D_{v}$ and $D_{v}^{*}$ are heavy, and v-hadronic decays such as $D_{v} \rightarrow \eta_{v}^{\prime} \omega_{v}$, $\eta_{v}^{\prime} \sigma_{v} \omega_{v}$, etc., are kinematically allowed. This can lead to final states with four or more jets and/or leptons, which may appear promptly or emerge from a displaced vertex anywhere in the detector, depending on the strength of the flavor-changing coupling. By contrast, in the $2 \mathrm{LF}$ regime, kinematics forbids v-hadronic decays of the $\pi_{v}^{+}$. The $\pi_{v}^{+}$instead decays to heavy-flavor $f \bar{f}$ or to $f \bar{f} \pi_{v}^{0}$. Its lifetime is much longer than that of the $\pi_{v}^{0}$, Eq. (11).

Existing Experimental Constraints: Cosmological constraints are minimal: v-strong interactions ensure that all v-hadrons annihilate efficiently to the lightest v-mesons, failing to do so only if there are asymmetries in conserved quantities such as v-baryon number or $C$-number. (Indeed, such asymmetries could permit stable v-mesons or v-baryons to be self-interacting dark matter.) Were all vmesons stable, they would form a cold gas and dominate over ordinary matter at temperatures somewhat below the scale $\Lambda_{v}$, which would ruin big-bang nucleosynthesis (BBN); but as long as one light $\mathrm{v}$-meson has a lifetime $\ll 1$ second, requiring $\Lambda_{v} \gtrsim 1 \mathrm{GeV}$, processes such as $\pi_{v}^{+} \pi_{v}^{-} \rightarrow \pi_{v}^{0} \pi_{v}^{0}$ followed by rapid $\pi_{v}^{0}$ decay (and analogous processes in the 1LF regime) reduce the density of v-pions exponentially before BBN occurs.

For models with light $\mathrm{v}$-hadrons, LEPI can place strong constraints. Results from $2 \times 10^{7} Z$ decays limit the branching fraction for $Z \rightarrow U \bar{U}, C \bar{C}$, via $Z-Z^{\prime}$ mixing, although the degree of constraint depends on the dominant final states. A rough estimate is given by treating the v-quarks as free:

$$
\Gamma_{Z \rightarrow U \bar{U}} \sim \frac{n_{v}}{6 \pi} \frac{g^{\prime 4}}{m_{Z^{\prime}}^{4}} Q_{H}^{2}\left(q_{+}^{2}+q_{-}^{2}\right) \frac{m_{Z}^{5}}{g_{1}^{2}+g_{2}^{2}}
$$

This gives a branching fraction, if $m_{Z^{\prime}} / g^{\prime}=10 \mathrm{TeV}$ and $n_{v}=3$, of about $10^{-7}$. (Note it will be enhanced if $m_{\omega_{v}} \sim$ $m_{Z}$.) Thus, if the v-hadronic decays of the $Z$ are easily distinguished from background, then $m_{Z^{\prime}} / g^{\prime}>10 \mathrm{TeV}$. Even then, LHC cross-sections for $U, C$ production for $m_{Z^{\prime}}=2.5 \mathrm{TeV}, g^{\prime}=0.25$, are of order $20 \mathrm{fb}$ (Fig. 2). Moreover, many models evade these constraints. In the $2 \mathrm{LF}$ regime, the decay $Z \rightarrow \pi_{v}^{0} \pi_{v}^{0}$ is forbidden, while $Z \rightarrow \pi_{v}^{+} \pi_{v}^{-}$is invisible, and the branching fraction for the partly visible decay $Z \rightarrow \pi_{v}^{+} \pi_{v}^{-} \pi_{v}^{0}$ is of order ten times smaller. The 1LF regime is more complex; multiobject final states with $b$ s have significant backgrounds, but a widely displaced vertex could reveal them easily. More detailed study is needed.

LEPII constraints are weaker than the bound (6) but may be important if that bound is evaded. As each experiment recorded $<1 \mathrm{fb}^{-1}$ of integrated luminosity, and as backgrounds to multi-jet and jets-plus-missingmomentum events are larger than at LEPI, the crosssection limit is probably weaker, and potentially much weaker, than $1 \mathrm{fb}$. The $e^{+} e^{-} \rightarrow Q \bar{Q}$ cross-section at 200 $\mathrm{GeV}$ is of order $1 \mathrm{fb}$ for $n_{v}=3$ and $m_{Z^{\prime}} / g^{\prime} \sim 6 \mathrm{TeV}$, though this requires more careful treatment, especially near the v-hadron resonance region.

Production and Signals at Hadron Colliders: Given these constraints, cross-sections at the LHC are typically in the 1-100 $\mathrm{fb}$ range, and $1 \mathrm{fb}$ or less at Tevatron, unless the LEP constraints are weaker due to kinematics or unobservable signals. Production of v-quark pairs at the

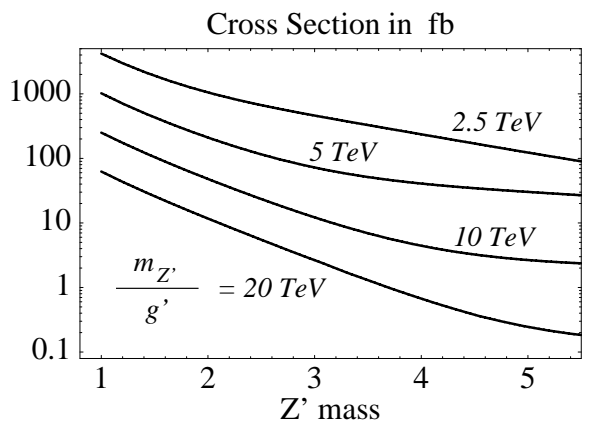

FIG. 2: Tree-level v-quark production cross-section in $\mathrm{fb}$ versus $m_{Z^{\prime}}$, for different $m_{Z^{\prime}} / g^{\prime}$; here $n_{v}=3, k=0, q_{+}=-2$, and $\Lambda_{v}, m_{U}, m_{C} \ll m_{Z^{\prime}}$.

LHC will occur at and below the $Z^{\prime}$ resonance. Since $q \bar{q} \rightarrow U \bar{U}$ is analogous to $e^{+} e^{-} \rightarrow q \bar{q}$, the production of v-hadrons recapitulates the physics at ADONE, SPEAR, VEPP and PETRA. At these experiments, pion multiplicities (for $E_{c m} \gg m_{\rho}$ ) averaged roughly $2 \ln E / m_{\pi}$, with considerable spread [14]. Events were spherical at $E \lesssim 3 \mathrm{GeV}$ and became increasingly two-jet-like as $E$ increased [15]. Thus LHC v-quark production will lead to multiple v-hadrons, with the ratio $m_{Z^{\prime}} / \Lambda_{v}$ determining the average multiplicity and whether the v-hadrons are distributed spherically, in two collimated $\mathrm{v}$-jets, or something in between.

For the $2 \mathrm{LF}$ regime, the dominant signal is multiple $b$-jet pairs (from $\pi_{v}^{0}$ decays) plus missing energy (from invisible v-hadrons), with a wide distribution in the number of $b$ jets and of the total missing energy (as in Fig. (3). The missing energy, muons from $b$ quarks, and occasional 
$\tau$ pairs may assist with triggering and event selection. If the $\pi_{v}^{0}$ is light, the jet pairs may be soft, may often merge, or be otherwise hard to identify, making triggering and event selection subtle; displaced vertices may assist in any discovery. If the $\pi_{v}^{0}$ is heavy, the jets will be harder, but the number of $b$ pairs may be smaller, decays will be prompt, and both QCD and $Z$ plus jets will be irreducible backgrounds. However, in this case LEP constraints are completely evaded and production cross-sections could be much larger.

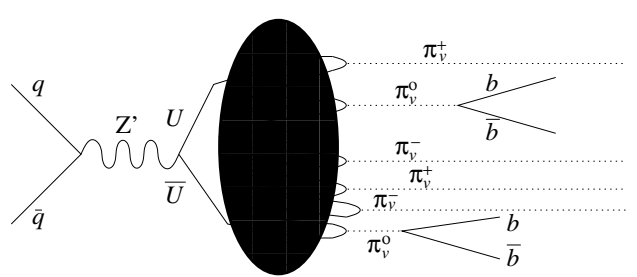

FIG. 3: A possible event in the two-light-flavor regime; note $\pi_{v}^{ \pm}$is electrically neutral and invisible.

The $1 \mathrm{LF}$ regime, with a greater variety of v-hadrons and final states, produces more lepton pairs. The $\omega_{v}$ will appear as a $\ell^{+} \ell^{-}$resonance; this will be drowned in Drell-Yan background unless events are required to have many $b$ s or an unusual displaced vertex. Especially interesting are the final states from $\sigma_{v}$ and $\sigma_{v}^{\prime}$, where $f \bar{f}$ pair emission is followed by an $\eta_{v}^{\prime}$ decay. One may observe $\sigma^{\prime} \rightarrow \mu^{+} \mu^{-} b \bar{b}$, with $m_{b \bar{b}}=m_{\eta_{v}^{\prime}}$ and $m_{\mu^{+} \mu^{-}}<$ $m_{\sigma_{v}^{\prime}}-m_{\eta_{v}^{\prime}}$. Even more spectacular decays are possible, with several objects emanating from a displaced vertex (or two), if the $C$ is unstable. One challenge is that lepton isolation may be subtle here; another is that displaced vertices may appear in the beampipe, the tracker, or even the calorimeter. Moreover, a given event may produce several v-hadrons, which can combine these distinctive signals into a busy and unusual event (as in Fig. 4), in which identification of jets may be challenging. As in the 2LF regime, large $\Lambda_{v}$ means fewer v-hadrons per event and fewer displaced vertices, but any jets and leptons are harder, and the LEP constraints on the total crosssection are weaker or absent.

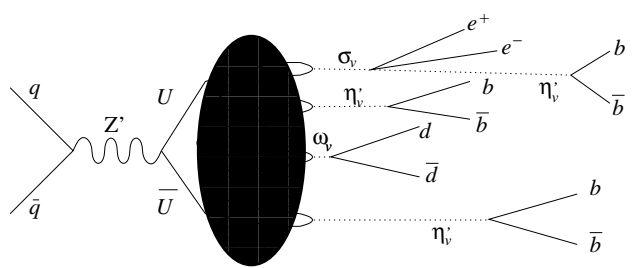

FIG. 4: A possible event in the one-light-flavor regime.

Let us add a few assorted remarks.

- The model we have chosen is a bit pessimistic [7], in that the $Z^{\prime}$ has large couplings to leptons (increasing LEP constraints) and small couplings to $u$ quarks (reducing Tevatron and LHC production rates.) The constraints quoted here are conservative; many models can have larger cross-sections at both LHC and the Tevatron.
- The high multiplicity of v-hadrons, especially for small $\Lambda_{v}$, has many implications. In events where most particles decay in the detector, jets and isolated leptons can be difficult to identify. Conversely, in models where the average v-hadron decays promptly, or in models with most decays outside the detector, the multiplicity increases the odds of seeing a straggler that decays with a visible vertex. It also enhances the possibility of detecting decays of long-lived v-hadrons (as might occur for unstable $C$, and would occur in the model mentioned below) by instrumenting the detector hall or a nearby cavern.

- Given (6), a GigaZ machine would likely be able to observe $Z$ decays to light v-hadrons. A high-luminosity $e^{+} e^{-}$collider could also study vector v-meson resonances and their $\mathrm{v}$-hadronic decay products.

- There has been interest in Higgs decays to multiple scalars, which in turn decay to heavy-flavor pairs [2, 4]. Our v-model may initially mimic this scenario, since the $2 \mathrm{LF}$ and $1 \mathrm{LF}$ regimes both have a (possibly light) pseudoscalar with $\operatorname{Br}\left(\pi_{v}, \eta_{v}^{\prime} \rightarrow f \bar{f}\right) \sim m_{f}^{2}$.

- We have taken the $N_{i}$ to be stable, but this need not be the case. Their striking decays in usual $Z^{\prime}$ models 7 ] would be further augmented by v-hadronic final states.

Higgs Mixing: Potentially of great importance is the effect of the mixing of $H$ with $\phi$, via a $|H|^{2}|\phi|^{2}$ coupling, along the same lines as [3]. This allows $g g \rightarrow h \rightarrow Q \bar{Q}$, which is unaffected by LEP constraints and can potentially increase the v-hadron production rate at the $\mathrm{LHC}$ and especially at the Tevatron. Decay modes for some v-hadrons may be affected. Kinematics permitting, the Higgs can even decay to v-hadrons. Though rare, these exotic Higgs decays could be so distinctive, if they have displaced vertices and/or leptons, as to possibly allow the Tevatron to discover the Higgs with its present data. This requires asking the right analysis questions, such as [16], though in a more systematic and comprehensive fashion. The masses, mixings and branching fractions of the $H$ and $\phi$ are very model-dependent; a separate study of these phenomena will be required.

Other Models: Other regimes of this theory, models with more v-quarks, and other $Z^{\prime}$ models will typically have similar phenomenology but differ in important details. (For instance, if $m_{U}, m_{C} \gg \Lambda_{v}$, the many stable glueballs will have longer lifetimes; many will decay outside the detector, and displaced vertices will be common.) $Z^{\prime}$ models with supersymmetry, a little higgs, extra dimensions, etc., would have additional diverse vphenomenology that we will not discuss here. Instead, to provide a wider perspective, we conclude with a class of models that generate qualitatively different phenomena.

Consider adding to the SM an $S U\left(n_{v}\right)$ gauge group, and particles $X, \bar{X}$ charged under both color and $S U\left(n_{v}\right)$, with $m_{X} \sim 0.5-3 \mathrm{TeV}$. The v-spectrum includes several metastable v-glueballs of mass $\sim \Lambda_{v}$ and various spins [17]. A loop of $X$ particles induces dimension-eight operators including $\mathcal{O}_{8} \equiv \operatorname{tr} G^{2} \operatorname{tr} G_{v}^{2}$, where $G\left(G_{v}\right)$ is the field strengths for gluons $g$ (v-gluons $g_{v}$.) All v-glueball states can decay through these operators; those that 
decay to $g g$ have lifetimes of order $\sim 8 \pi M_{X}^{8} / \alpha_{s}^{2} \alpha_{v}^{2} \Lambda_{v}^{9}$. which for $m_{X} \sim 1 \mathrm{TeV}, \Lambda \sim 100 \mathrm{GeV}$ are in the range 1 psec -1 nsec. Glueballs decaying to $g g g$ live at least $10^{3}$ times longer. Heavy-quark and lepton pairs are absent, making identification of the signal challenging. However, given the range of glueball lifetimes, displaced vertices are not unlikely. Note there are no constraints from LEP; decay of cosmic confining strings may be a cosmological problem, but can be evaded if necessary.

While $\mathcal{O}_{8}$ allows v-glueballs to be produced through $g g \rightarrow g_{v} g_{v}, g g \rightarrow g g_{v} g_{v}$, etc., pair production of $X \bar{X}$ also suffices and has a large cross section. The phenomenology of these events can vary widely. Consider three possibilities, among many others:

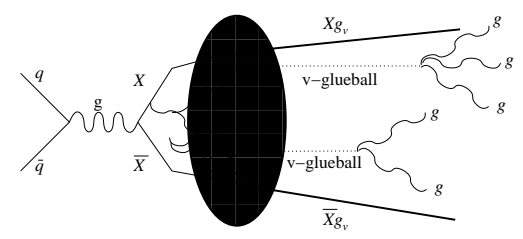

FIG. 5: $X \bar{X}$ pair production with associated v-glueballs, for a stable $X$ in the adjoint of $G_{v}$; the $X g_{v}$ bound state resembles a stable gluino.

- If $X$ is an adjoint of $S U\left(n_{v}\right)$ and decays immediately to a v-gluon plus one (or more) SM quarks or gluons, then each $X$ will decay to one (or more) hard jets recoiling against v-glueballs (each giving softer jets).

- If $X$ is a stable triplet (or octet) of color and an adjoint of $S U\left(n_{v}\right)$, it will bind to a v-gluon, forming a composite color-triplet (or octet.) This composite $\left(X g_{v}\right)$ can be distinguished from a stable quark (or gluino) by the multiple v-glueballs that will often accompany it, e.g. in $q \bar{q} \rightarrow X \bar{X} g_{v}$ events (Fig. 5 ).

- If $X$ is stable and in the $n_{v}$ of $S U\left(n_{v}\right)$, then $X$ and $\bar{X}$ will be bound by a v-confining flux tube. The bound system will lose energy by gluon emission (giving a soft jet) or emission of v-glueballs (giving multiple jets); eventually $X$ and $\bar{X}$ will annihilate to gluons (giving two or more ultra-hard jets) or to v-gluons (generating several v-glueballs.)

Final Remarks: Clearly the range of possible vphenomena is wide. Can it be observed? Whether backgrounds are large depends strongly on parameters. In some cases, trigger efficiencies may be an issue; in almost all cases, event selection for an analysis is a complex matter. High-multiplicity events with many soft jets are especially challenging; defining jets and isolated leptons may be problematic. The phenomenology is rich and complex and will require novel simulation techniques. We hope this work will stimulate discussion of how to ensure that physics of this type does not go undetected.

We thank P. Langcker, H. Lubatti, A. Nelson, S. Sharpe, M. Shifman, P. Skands and G. Watts for helpful assistance. MJS thanks T. Han, P. Langacker and K. Freese for discussions of related topics in 2001. Some of these ideas were presented at the Atlas Muon Workshop at U. Washington, July 2004. This work was supported by U.S. Department of Energy grants DE-FG0300ER41132 (KZ) and DE-FG02-96ER40956 (MJS).
[1] N. Arkani-Hamed and S. Dimopoulos, JHEP 0506, 073 (2005) arXiv:hep-th/0405159; A. Arvanitaki, S. Dimopoulos, A. Pierce, S. Rajendran and J. Wacker, arXiv:hep-ph/0506242

[2] U. Ellwanger, J. F. Gunion, C. Hugonie and S. Moretti, arXiv:hep-ph/0305109 arXiv:hep-ph/0401228 R. Dermisek and J. F. Gunion, next Phys. Rev. Lett. 95, 041801 (2005) arXiv:hep-ph/0502105. U. Ellwanger, J. F. Gunion and C. Hugonie, JHEP 0507, 041 (2005) arXiv:hep-ph/0503203; R. Dermisek and J. F. Gunion, arXiv:hep-ph/0510322

[3] R. Schabinger and J. D. Wells, Phys. Rev. D 72, 093007 (2005) arXiv:hep-ph/0509209.

[4] S. Chang, P. J. Fox and N. Weiner, arXiv:hep-ph/0511250

[5] V. Barger, P. Langacker, H. S. Lee and G. Shaughnessy, arXiv:hep-ph/0603247

[6] See for example M. Cvetic, P. Langacker and G. Shiu, Phys. Rev. D 66, 066004 (2002) arXiv:hep-ph/0205252, and references therein; N. Arkani-Hamed, S. Dimopoulos and S. Kachru, arXiv:hep-th/0501082

[7] R. W. Robinett and J. L. Rosner, Phys. Rev. D 25, 3036 (1982) [Erratum-ibid. D 27, 679 (1983)]; P. Langacker, R. W. Robinett and J. L. Rosner, Phys. Rev. D 30, 1470 (1984).
[8] F. del Aguila, G. A. Blair, M. Daniel and G. G. Ross, Nucl. Phys. B 283, 50 (1987); F. del Aguila, G. D. Coughlan and M. Quiros, Nucl. Phys. B 307, 633 (1988) [Erratum-ibid. B 312, 751 (1989)]; K. R. Dienes, C. F. Kolda and J. March-Russell, Nucl. Phys. B 492, 104 (1997) arXiv:hep-ph/9610479; K. S. Babu, C. F. Kolda and J. March-Russell, Phys. Rev. D 57, 6788 (1998) arXiv:hep-ph/9710441; T. G. Rizzo, Phys. Rev. D 59, 015020 (1999) arXiv:hep-ph/9806397.

[9] E. Witten, Nucl. Phys. B 149, 285 (1979); Nucl. Phys. B 156, 269 (1979); G. Veneziano, Nucl. Phys. B 159, 213 (1979).

[10] M. J. Strassler, arXiv:hep-th/0104032

[11] A. Armoni, M. Shifman and G. Veneziano, Nucl. Phys. B 667, 170 (2003) arXiv:hep-th/0302163; Phys. Rev. D 71, 045015 (2005) arXiv:hep-th/0412203.

[12] A. Armoni, M. Shifman and G. Veneziano, Phys. Rev. Lett. 91, 191601 (2003) arXiv:hep-th/0307097.

[13] F. Sannino and M. Shifman, Phys. Rev. D 69, 125004 (2004) arXiv:hep-th/0309252; A. Gorsky and M. Shifman, Phys. Rev. D 71, 025009 (2005) arXiv:hep-th/0410099; A. Armoni and E. Imeroni, Phys. Lett. B 631, 192 (2005) arXiv:hep-th/0508107; M. Shifman, private communication.

[14] M. Spinetti, LNF-79/25-P Presented at 14th Rencontre 
de Moriond, Les Arcs, France, Mar 11-23, 1979; C. Bacci et al., Nucl. Phys. B 184, 31 (1981); J. Siegrist et al., Phys. Rev. D 26, 969 (1982).

[15] G. Hanson et al., Phys. Rev. Lett. 35 (1975) 1609.
[16] DZero note 5023-CONF, preliminary result, March 2005.

[17] C. J. Morningstar and M. J. Peardon, Phys. Rev. D 60, 034509 (1999) arXiv:hep-lat/9901004. 